\title{
Introduction to How to Lead Academic
}

\section{Departments Successfully}

Ask any group of academic heads of departments about their current projects and their current challenges, and a surprisingly large range of responses will follow. In preparation for an executive teaching program aimed at university heads, two of this book's editors collected feedback on exactly this. From a group of 20 departmental leaders, we received a total of 30 different projects and challenges. What was particularly interesting was not just the number of separate responses but also their diversity.

We will not list all the responses. However, some sense of this diversity can be taken from just a few examples:

- Retention and recruitment;

- Research underperformance;

- Academic citizenship;

- Own research time;

- Educational reform and development;

- Institutional accreditation/quality assurance;

- Organizational change;

- Trust and collaboration.

The list went on, citing generational shifts, working with industry, scientific impact, and external funding. Of course, we did our best to cluster these topics, but it is difficult to squeeze together international collaboration, time management, and societal challenges, for example, under one heading.

The point - which these 20 department heads agreed with, once they saw the entire list - is that each challenge is recognizable, but together, they look formidable. This moment sowed the seed and inspiration for this collection. In a practical sense, we uncovered a key question: How can we use the experience of heads of department, and others with relevant expertise, to help current academic managers increase their own efficiency and effectiveness? For those who are new to (or just curious about) this crucial position, how can we help them understand more? And for experienced academic leaders, how can we provide new ideas and reflections - and even some inspiration? Projects and challenges are unlikely ever to go away completely, nor is that a relevant goal. But how can we, as academic leaders, improve our knowledge and practices so that we can deal with them successfully?

In subsequent discussions with participants in the executive course, some additional shared challenges emerged. Roughly, we can group them under four headings. First, strategic chal- 
lenges confront every departmental leader. Typically, they are not either/or questions but related to how to tackle multiple issues at the same time. Thus, leaders must balance between:

- Research and teaching requirements;

- National and international requirements;

- Research quality and quantity;

- Senior and junior staff;

- Top-down and bottom-up management styles.

Academic leaders note they cannot focus on just one "bottom line" but instead must deal with multiple factors, in what can become a messy process that appears to lack clear prioritization.

Second, these difficulties lead into the next set of issues, pertaining to the roles and responsibilities of the department head. Across the range of inputs, we derived some key requirements related to this task. The head of department must ensure:

- The department's contribution to the overall university research strategy;

- The long-term development of the department's main research, educational, and dissemination activities;

- Good internal working relations, based on inclusion, team spirit, and career development;

- Efficient coordination among staff at different career stages and with different administrative and academic competences.

The general point here is that good leadership must be exercised across several groups, including research staff and teachers, administrative personnel, and external stakeholders.

Third, there are leadership challenges associated with being head of department. For many new leaders, these represent the toughest challenges of all. If one is promoted into this position, how does one then take over responsibility for one's colleagues? If one is hired from the outside, how does one gain authority among the local academic and administrative community? Why would anyone be led by you? Put simply, we can think of this as:

- Leading upward to deal with senior managers, including deans and presidents;

- Leading downward to work with the staff over whom one has formal responsibility;

- Leading out among external stakeholders and collaborators;

- Leading across by making connections with heads of department and other internal colleagues;

- Leading oneself by working constructively with one's own strengths and weaknesses.

Fourth, notably, the role of the department head varies across contexts. In some countries, for example, department heads are classed as administrative personnel, even if they must have academic qualifications in order to be appointed. In other settings, a rotating leadership tradition requires each professor to take a turn, sometimes for a relatively short period of time. Even within a country, different departments and universities vary substantially in their size, complexity, and organizational culture.

Each one of us also has our own motivation for the position. For some, this will be a (hopefully) rewarding experience for a fixed period. Others might be considering the position of department head as one stepping-stone on a career path leading to the role of dean, vice 
president or university president - and even beyond. In the following chapters, we try to take account of these different contextual and personal factors.

Even as we specify these four categories of challenges, we also acknowledge that the job is never static. Issues come and go. However, some questions - including those pertaining to diversity management, social inclusion, ethics, and integrity - have grown so much in importance that they are unlikely to fade. In this sense, we also are reminded that the university might seem like a world unto itself, but it both reflects and responds to broader societal developments and changes. One other example of this is the increasing use of indicators, both across different staffing levels and as applied to leaders themselves. There can be benefits here, but many university leaders experience performance measurement as adding to their work pressures.

We think we have made the basic point that academic leadership is not the easiest task. As the chapters of this book explore in considerable depth, the range and diversity of challenges can be daunting, especially to a new leader, but also to those who have been in the position for some time. The old cliché about herding cats comes to mind. As several contributors argue, an over-directive, top-down leadership style is unlikely to succeed in most academic settings.

At this point, current departmental leaders and those thinking of accepting the task might be tempted to stop reading. If the challenges are so great, why would anyone want to take on such a role? Why bother to read further in this book? We offer three arguments for doing so, all of which flow through the subsequent chapters.

First, departments (or schools, institutes) are the foundation on which universities are built. When asked about their work environment, most academics describe their "home" department, whereas the university seems more distant from their everyday professional life. Thus, the departmental setting is extremely important, and in turn, the department head is extremely important.

Our collective experience of working in and around university departments strongly indicates that the performance of a department head can make a huge difference, whether in shaping a positive, inspirational setting or (as can happen with remarkable speed) in sending a negative message of limited opportunities and restricted possibilities. Sometimes, the work of a departmental head is invisible and taken for granted. Nevertheless, the argument throughout the following chapters is that the potential of each department head to make a difference cannot be underestimated.

Second, as our previous discussion of projects and challenges reveals, serving as a head of department can be demanding. It provokes new personal and intellectual demands. However, it also offers a unique opportunity to see the academic world in a fresh light, viewing the university from the perspective of the whole, not just one's own point of view. The change in perspective can be startling, unsettling, and downright tiring. Yet it also can be a tremendous opportunity to develop new skills and learn new things (not least, about oneself).

Just as one example, a common experience is that one does not stand alone as a leader. Instead, successful heads of department operate within a network of colleagues, with different positions, competences, and attributes. For those used to working alone, or perhaps as part of a very small group of teachers and researchers, such teamwork can be surprising and difficult to embrace. It can also be a source of great satisfaction and consolation when (as inevitably happens) the best prepared plans begin to crumble. 
Third, we can learn from experience. In general, the chapters that follow do not offer a how-to guide or quick fix. Instead, many contributors have taken this opportunity to make sense of their own experience and offer the kinds of advice that they likely would have appreciated when they were starting their own personal leadership journey.

The ensuing chapters are grouped by broad themes, in what we hope is a coherent fashion. But we encouraged the contributors to express themselves in their own way. This diversity of approaches ideally makes the book engaging but also illustrates the central point that no recipe or formula for success exists. There are general lessons to learn, but no two leaders will follow exactly the same blueprint.

The book title, How to Lead Academic Departments Successfully, suggests though that beyond the diverse challenges of leadership, we will also cover its successes. Just as there is no exclusive bottom line, success takes different forms. Leadership involves a choice of focus and key goals, which requires asking about what is most important in any specific setting and what can, at least relatively, be assigned less emphasis.

The following chapters present varied approaches to success, both personally and in organizational terms. Together, they provide a practical, and hopefully stimulating, guide to success as a practicing or aspiring departmental head. We also hope this book is instructive for readers who want to understand how this crucial role in academia operates. Our experience, as editors, is that each contributor has introduced important new dimensions of this role, thereby showing us how much we still have to learn about this fascinating, and we would say crucial, leadership position in some of our most important institutions.

\section{PART I: CHALLENGES OF BEING AN ACADEMIC LEADER}

As mentioned in the introduction, the challenges that confront academic leaders in practice are numerous, particularly considering the developments taking place in academia but also what is happening in the external environment that affects universities. When asked whether it is more difficult or complex to be an academic leader today, compared with 20 years ago, the answer is not simple though; rather, because the context of leadership is constantly evolving, the best response is that "it is different." Furthermore, because there is no recipe for how to be a leader, whether in academic settings or in the corporate world, leadership always must be situational and aligned with followers. Leadership is not a mechanical device but a way to encourage each person's professional and personal talent in orchestrating a group of different actors and personalities. In the first section, three contributions thus highlight key issues, challenges, and rewards of being an academic leader, together with good advice for how to demonstrate successful leadership.

For example, Poulfelt challenges the myth that academics do not want to be managed or led. Rather, they require a particular kind of management, which eliminates the possibility of applying out-of-the-box solutions. This discussion "On the Particular Challenges of Managing Professionals" presents eight leadership virtues for academic settings, based on leadership theory and informed by the author's observations gained from four decades of experience with academic leadership. These virtues should inform leaders' daily practice, to increase their management success rate.

As Moore argues, because no perfect method exists for how to prepare academic leaders to helm the department, they encounter perpetual surprises, discoveries, and work-in-progress. 
In "Relevant Leadership: The Dynamic Equilibrium of Managing and Leading Academic Departments," the author proposes that department chairs are like frontline workers, required to assume multiple roles, responsibilities, and functions, and those functions often compete, collide, or contradict one another. To train and survive, while also learning how to do the job, effective leaders need to embrace the dynamic equilibria; this chapter offers some suggestions for how.

Similarly, Robson argues that a successful leader recognizes and heeds the tensions that arise across inevitable faultlines, at the business school and institutional level. Taking a dialectical view on "The Role of Academic Leaders of a Business School: An Internal Tensions Perspective," Robson identifies three specific faultlines in business schools: managerialism/ optimization versus entrepreneurialism/creativity; the individual versus the collective; and research versus teaching. Some leaders seek to rock the proverbial boat by introducing extreme policies, but best practices allow conflicting values to coexist within a broader equilibrium, which helps ensure institutional stability and success. The chapter therefore offers some ideas for how leaders might find some balance at the three identified faultlines.

\section{PART II: TRANSFORMATIONAL AND PERFORMANCE LEADERSHIP}

Leadership is not static; it occurs in environments that are constantly developing, sometimes very turbulently. This situation imposes pressures on leaders to ensure the organizational unit, whether a research group, a department, or a university, copes with the complexity and changes productively. Such leadership often is referred to as transformational, a form of leadership that emphasizes the capability to make meaningful changes that set the course of the organization and secure engagement and acceptance among most employees. It sounds easy. In practice, it is very demanding, particularly because the transformational leadership agenda requires a focus on performance at all levels - university, faculties, departments, and individual researchers. But such an intense focus on performance also creates new leadership challenges, including the need to balance autonomy with control. Therefore, this second section offers five contributions pertaining to the leadership roles and tasks required to secure valuable transformations and productive performance, by providing observations from the field, new insights, and valuable advice.

To start this section, Busk J. and Barlebo Rasmussen ask, what will it mean to be the head of department in the future? They note historical trends that suggest the answer will reflect contemporary perceptions of science in future society. Whereas in the recent past, the head of department mainly served a collective of autonomous faculty members, and then became something like an overseer managing a research factory, today they regard "The Head of Department as the Key Transformational Leader." Recognizing that the relationship between science and society continually changes, they identify growing demands for research with societal impact, which in turn means that the university's organization, strategy, and management must shift to meet them. As a key transformational leader, the head of department can lead this transformation and take on practical, meaningful management roles.

Kitchener agrees with the notion that society demands new roles of the university, which must ensure that its pursuit of accreditation or revenue does not distract it from its primary, societal purpose. He thus recommends "Leading with Purpose: Developing the First Business 
School for Public Good." With a personal account of efforts to achieve purposeful change at Cardiff Business School (United Kingdom), the author highlights the urgent need for business schools to undergo repurposing. In line with corporate purpose literature, three approaches can promote the development of purposeful organizations: a chief purpose officer, purpose statements, and multi-capital reporting. In describing the applications of these approaches, this chapter explains how Cardiff became the first business school to focus on enhancing the public good and thus offers insights for how other academic leaders might lead a similar repurposing.

Taking a broad view on "Leading Academic Departments," Goffee and Jones propose three fundamental axioms of leadership: it is contextual, relational, and non-hierarchical. But in academic work contexts, filled with "clever" academics, leadership must take unique forms to support their success. In particular, leaders must balance benevolent guardianship against discipline. In the effort to "be yourself, more, with skill," they also need to find ways to engage in authentic self-expression while also connecting successfully with others.

Continuing the focus on managing academics, Werr and Einola ask "Individual Performance Management: Enabler or Threat to Academic Performance?" To ensure the quality and quantity of academic output, universities often ask department heads to manage members' individual performance and conduct systematic performance reviews. But traditional performance management systems and appraisals do not match well with academic contexts. The middle managers have little power; the employees (academics) naturally exhibit multiple loyalties and demand freedom and autonomy. Noting emerging trends and research evidence about the consequences of performance management practices, this chapter offers several suggestions for adapting individual performance management methods to university settings.

Another option for adapting and applying external concepts to academic settings, as detailed in "Deploying Systems Thinking to Create a 'Triple-Crown' Business School," relies on critical systems thinking. Jackson provides an account of his experiences as the first dean of a UK business school that achieved remarkable progress between 1999 and 2011. Following from its foundation, when Jackson left in 2011, the school had hired 165 staff, attracted 3,500 students, and earned $£ 28$ million annually. With an excellent reputation for teaching, research, and reach, it had gained three key accreditations and a ranking among the top $1 \%$ of business schools worldwide. As Jackson describes it, systems thinking, and particularly critical systems thinking, was essential to bringing about this success.

\section{PART III: INCLUSIVITY, TEAM SPIRIT AND CAREER DEVELOPMENT}

Among the diverse, relevant topics that find their way onto the agenda of academic leaders, dealing with conflicts represents a particularly complex theme. It arises for leaders of research groups and departments and requires consideration of different factors, including professionalism, rationalities, and emotions. Limiting the amount of conflict and resolving those that arise in a constructive way can ensure engagement, energy, and a team spirit. A team spirit in turn can have tremendous impacts on achieving optimal outcomes. Even though most academic institutions claim they want to attract the best academic researchers and staff, achieving that goal also represents a critical challenge, especially when addressed in conjunction with diversity goals. Therefore, this section, with five contributions, offers case stories, examples, and recommendations for dealing with these issues in practice. 
The title of the first chapter in this section offers a clear caution: "'Don't Think You Can Be Everyone's Friend': Dealing with Conflict in an Academic Department." The author contests conventional views of conflict as an inevitable characteristic of academia that demands a rational, planned approach. Rather, the complexities linked to conflict in practice give rise to three stories, related broadly to interpersonal conflict, departmental conflict, and institutional conflict. Irwin argues that conflicts often cannot be neatly classified or defined but may encompass various directions, sometimes over considerable time; with personal insights, this author offers some further perspectives and advice.

The next title is evocative too: in "Smells like Team Spirit: A User's Guide for Department Heads," Kjær proposes ways to leverage team spirit, defined as a sense of belonging among members of a department, using concrete examples from his experience as the head of a department in a Danish university. He lists several relevant tools, reflecting different dimensions of managerial practice, to help departmental members make sense of and connect to the collective entity, as well as some dilemmas and paradoxes that arise in this process.

Even if a leader's normative framework centers on fairness and equality, as is the case for the author of this article, achieving inclusion and diversity can be challenging. Horst details three factors in "Now You See It': Gender, Inclusion and Diversity" that emerged from her efforts to do so: structural decoupling, which reflects disconnects between diversity and other required managerial activities; unconscious bias, pertaining to implicit expectations of others; and recruitment. Using this framework, Horst also details what should have done differently. Other leaders can take inspiration from this to inform and improve their diversity efforts.

In Andersen's chapter, "Leading Faculty as Teachers," the focus is on two elements of leading academic departments that must offer both teaching and research, even though these two projects evoke different valuations. First, leaders must lead faculty in their career progression as teachers. Second, they must help faculty manage their workload distributions when allocating teaching duties. As heads of departments, these leaders have unique mediating functions, between institutional and faculty perspectives on the importance of teaching.

Another critical factor for leading faculty members is ensuring their integration into their departments, to achieve "Inclusive Onboarding in Academic Departments." By exploring the lived experiences of new faculty members in an academic department in a business school environment, Petzer, Kleyn, and Ruiters seek to understand the perceptions that new faculty develop, in terms of feeling included and assimilated. The qualitative data, gathered from new, full-time faculty members of a South African business school, reveal critical incidents that produce positive inclusion experiences, but also some evidence of exclusion. These incidents in turn indicate which actions heads of departments should undertake to guarantee inclusive faculty onboarding.

\section{PART IV: BUILDING, LEADING AND FUNDING RESEARCH GROUPS}

Strong and distinct research profiles can enhance a university's standing and competitive appeal, but research is rarely performed in isolation. High-profile research groups are critical, but to establish them, universities need methods for attracting and retaining the best talents and scholars, as well as sufficient external funding for those groups. Such efforts inevitably involve dilemmas, as the three contributions in this section acknowledge. In addition to high- 
lighting fundamental issues associated with building and leading research units, from research and practice perspectives, these chapters continue to offer leadership advice.

When it comes to "Building Research Groups," Lindgreen, Di Benedetto, Brodie, and Naudé propose that attracting the top research talent requires a successful research environment first, which promises academic researchers that they will be able to produce a sustainable research stream. In turn, this environment demands several antecedents, including a clear business school research strategy, leadership, governance, and policy. To illustrate the conditions that can support the long-term success of research programs, the authors elaborate on two active research institutions: the Industrial Marketing and Purchasing (IMP) Group and the Contemporary Marketing Practices (CMP) Group. These examples in turn reveal which environmental conditions are most conducive to sustainable business school research, as well as the gatekeeping role of journal editors.

The Bologna initiative aims to standardize higher education policies. Its implementation by universities in Denmark has been relatively substantial, such that they offer a leading example of the dilemmas that this legislation has created. Copenhagen Business School provides an illustration in "Dilemmas in University Management: The Case of Copenhagen Business School." Mik-Meyer draws on her experience as Vice Dean of Research to highlight the benefits and detriments of using key performance indicators of research quality.

Another international issue is external funding, which departments and universities increasingly must seek, such that this pursuit shapes their organizational discourses and research practices. Hofeldt argues that departments are like "shop floors," where organizational cultures of support and empowerment can be established. Hofeldt phrases the question in terms of "Pathways to External Funding at Departments: How to Strengthen a Change of Culture by Empowerment, Supportive Organizing, and Leadership?" By also analyzing barriers to change, erected by both researchers and department managers, the author derives an integral, concrete, three-level approach, spanning the personal, the organizational, and the managerial levels. He also proposes a new project design tool, the Project Model Canvas, which reveals both individual-level competencies and strategic support and leadership frameworks at the departmental and university levels.

\section{PART V: COLLABORATION WITH OTHER DISCIPLINES AND PRACTITIONERS}

The three contributions in this section address cross-disciplinarity and collaborations, as increasingly popular methods for dealing with complex problems that pertain to multiple disciplines. Furthermore, many universities actively work to expand their community ties to exploit the notable benefits that can accrue from involving external stakeholders, such as corporations, public sector organizations, or nonprofit actors in research projects. Yet these developments also put additional pressure on academic leaders to develop new interaction and collaboration modes; working with practitioners in particular often requires a different approach on both sides, as Di Benedetto, Lindgreen, Storgaard, and Højbjerg Clarke describe in "Collaborating with Practitioners." Their meta-perspective on collaborations between academics and businesspeople suggests ways to encourage them. In particular, they show that academics often take the university perspective, intuitively and implicitly, when talking about collaborative research. They consider why, how, and what questions. But an outside, business 
perspective on the same collaboration might focus more on innovations. Comparing these two perspectives reveals not just their differences but also the learning opportunities they create.

Taking a finer-grained view, Jeppesen reviews a collaboration involving oil and gas industry firms and the Danish Hydrocarbon Research and Technology Centre at the Technical University of Denmark. In so doing, he proposes a new model for cross-disciplinary collaborations, as a response to the challenges that arose during the first six years of the collaboration. That is, "Leading Academics in a Public-Private Partnership: Balancing Value and Performance-Based Leadership in Times of (Climate) Change" outlines how, to deal with the difficulties, they fundamentally rejected a classic academic organization and embraced a closely managed, open collaboration model, then adapted the research and innovation strategy to reflect industrial and societal needs as they shifted. To do so, the project participants relied on professional portfolio and project management approaches, as well as leaders that took care to balance value against performance.

Similarly, business-to-business research is inherently cross-disciplinary, because theories about business relationships, systems, and markets come from economics, psychology, sociology, and management disciplines. When "Undertaking Cross-Disciplinary Research," academic researchers therefore need to overcome functional silos, which likely create differences in the collaborators' incentives, culture, terminology, and jargon, as well as a risk of opportunistic or counterproductive behaviors. Lindgreen, Di Benedetto, Brodie, and van der Borgh therefore seek recommendations for how to undertake cross-disciplinary research that advances the business-to-business literature stream, namely, by elaborating on theorizing processes and actively breaking down cross-disciplinary boundaries.

\section{PART VI: LEADERSHIP IN DIFFERENT CONTEXTS}

Leadership is contextually bounded; it must be exercised in a way that reflects the needs and situations of each specific organization or group. This is not to say that leaders cannot learn from others' examples, but they still must be able to transcend and translate these insights into specific contextual and organizational actions, as a form of contextual intelligence. The contributions in this section similarly call for contextual leadership, such that the lessons they provide should be translated to reflect readers' own leadership environment.

For Fischbacher-Smith, the dominant context reflects a systems approach. Calling for "Framing Business Schools as a Socio-Technical System: Issues around Complexity and Emergence," this chapter frames each school as a socio-technical system that contains various elements, reflecting its design. A change to the designed state then can push the system toward a new state, as can alterations to the operating environment. In combination, such shifts might generate control fractures that require attention from leaders and managers. Another issue arises from competing views of core processes, such that various members of the system generate sometimes incompatible demands.

For Peters, the context with the greatest relevance is the internal institution and the range of activities it must undertake. For example, undergraduate and some masters programs are more like consumer markets, but executive education often entails a business-to-business transaction. In "Business School Leadership in an Era of Change and Uncertainty: Complex Structures, Executive Education and Accreditation," Peters notes the different paths to market, delivery modes, staffing approaches, and skill sets required by these programs. For example, 
many executive education providers confront severe financial limitations, prompting mergers, acquisitions, and restructuring. In the parent university, similar pressures are leading to centralization initiatives that challenge business schools' control over their own destinies. Such changes ultimately will require accreditation bodies to review their expectations about institutional autonomy to develop more realistic requirements.

Finally, for Eskildsen and Obel, Denmark provides a meaningful context. Whereas academic institutions had remained basically stable for hundreds of years, a 1970 law prompted a long-running wave of dramatic, significant changes. As they recount in "Academic Leadership: The Danish Case," the new rules created new governance structures, financing models, incentives, academic roles, and leadership conditions. With a historical view on their own academic leadership experience, they use a theoretical framework to describe these changes and their implications for academic leadership, from 1968 to 2020.

\section{PART VII: PERSONAL LEADERSHIP REFLECTIONS}

Finally, the three contributions in this section offer personal observations and reflections on being an academic leader. These authors take readers to the frontlines of the leadership battle and offer hearty food for thought for anyone involved in leadership, whether as a newly appointed head or someone with vast experience.

Cummings cites 21 years of experience as department chair to offer advice for performing this role, based on six "Responsibilities of the Department Chair: Lessons from the Frontline": (1) norming, (2) strategizing, (3) guiding, (4) complying, (5) developing, and (6) behaving. This list, along with concrete examples, provides a useful summary of topics worth considering by department chairs.

Van Bruggen's reflections and insights on "How to Lead an Academic Marketing Department: Some Personal Observations and Reflections" combine into what he calls a "big plea": keep things simple, let go of control, and cherish other people's power and creativity by giving them freedom to work on what they really care about. Accordingly, van Bruggen argues that an academic leader's main purpose is to challenge, encourage, trust, and facilitate, so even if incentives and extrinsic rewards are nice, helping followers find their intrinsic motivations and realize their potential is really the key.

Finally, Møllgaard describes the journey "From Head to Dean: Academic Leadership" by outlining its purpose. That is, the purpose of leadership is to prioritize the activities of those they lead, over their own. Such purpose (or rationale for action) is not just essential but also essential to communicate. But leaders also confront dual loyalties, to protect the department from senior management and to embrace senior management when sharing information with the department. With good training, a dean can meet seven prominent challenges: selecting the right department heads, delegating managerial tasks, coaching, facilitating dialogue about departmental performance, allocating resources across departments, managing boundaries between them, and co-creating a purpose.

\section{CLOSING REMARKS}

We extend a special thanks to Edward Elgar and its staff, who have been most helpful throughout this entire process. Equally, we warmly thank our contributors with whom we 
have worked closely. They have exhibited the desire to share their knowledge and experience with this book's readers - and a willingness to put forward their views for possible challenge by their peers. We hope that this compendium of chapters and themes stimulates and supports colleagues in their teaching, learning, and leadership development. 
'Few things - if any - are more important to universities than departments and their capacity to provide the organizational framework for successful scholarly collectives. Hence, department heads are key personnel in any academic institution. How to Lead Academic Departments Successfully offers multiple perspectives and insights into the art and craft of being an effective head of department. The book holds a unique collection of astute and inspirational chapters and the volume should be of great interest to anyone with an interest in academic leadership.' Jens Ringsmose, Southern University Denmark

'Few colleagues plan (and prepare) to be an academic leader at the start of their career. But, almost inevitably, many of us will be asked to take up a major leadership role at some point, and often unexpectedly. Managing professionals within a distinct organisational climate, with unique practices and politics, against the backdrop of a dynamic marketplace is no small matter. It is, in fact, often a sink-or-swim experience. But, have no fear, How to Lead Academic Departments Successfully is

here. The book is a rich source of information that offers actionable advice and perhaps comfort for those of us who are about to face the challenges of academic leadership or are trying their very best at it every day. The book's content provides lucid and eclectic answers to the question posed in its title. It will be an inspiring companion of travel for any colleague on a leadership journey.'

Ko de Ruyter, King's College London, UK

'This is an important book addressing many central issues regarding how to effectively lead academic departments, thereby also pointing towards what might become the business school/academic institution of the future. The area of managing academic institutions is largely both under-researched as well as being largely void in the literature. The book makes a significant contribution by filling much of this gap. Specifically, it is a major message of the book that firm, clear leadership and strategic vision is called for by department heads (as well as deans and rectors), and thus that the widely shared view that professors should be left to themselves may not be enough!'

Peter Lorange, IMD and Lorange Network

'Being head of an academic department has not become less challenging in the 21st century with growing and changing demands on the universities from society. Drawing on the collective knowledge and experience of leaders and scholars from the university sector, this book provides a treasure chest of perspectives, ideas and real-life experiences that are both highly relevant and very useful for any current (or future) head of department - and for anyone with an interest in leadership in academia.'

Nikolaj Malchow Møller, Copenhagen Business School, Denmark

'Academic life sometimes appears to be a riddle hidden inside an enigma. Universities should be relevant to the local community, attractive to global students, visible in the finest academic journals, collegial in style yet run by accountable leaders. This hybridized academy is in urgent need of a guide from which the frustrated insider can get consolation and advice. This is the book, drawing on personal experience and a wide range of sources filtered through the analytic lens of seasoned social scientists. A twist of humour makes it even more essential, and it will be of equal importance to the higher education scholar and to the professional in need of perspectives.'

Mats Benner, Lund University, Sweden 